\title{
Application of perturbation theory to a P-wave eikonal equation in orthorhombic media
}

\author{
Alexey Stovas ${ }^{1}$, Nabil Masmoudi ${ }^{2}$, and Tariq Alkhalifah ${ }^{2}$
}

\begin{abstract}
The P-wave eikonal equation for orthorhombic (ORT) anisotropic media is a highly nonlinear partial differential equation requiring the solution of a sixth-order polynomial to obtain traveltimes, resulting in complex and time-consuming numerical solutions. To alleviate this complexity, we approximate the solution of this equation by applying a multiparametric perturbation approach. We also investigated the sensitivity of traveltime surfaces in ORT media with respect to three anelliptic parameters. As a result, a simple and accurate P-wave traveltime approximation valid for ORT media was derived. Two different possible anelliptic parameterizations were compared. One of the parameterizations includes anelliptic parameters defined at zero offset: $\eta_{1}, \eta_{2}$, and $\eta_{x y}$. Another parameterization includes anelliptic parameters defined for all symmetry planes: $\eta_{1}, \eta_{2}$, and $\eta_{3}$. The azimuthal behavior of sensitivity coefficients with different parameterizations was used to analyze the crosstalk between anelliptic parameters.
\end{abstract}

\section{INTRODUCTION}

The seismic model with orthorhombic (ORT) anisotropy is recognized as the most encompassing, and yet somewhat practical, anisotropic representation of earth's complexity. It combines the natural vertical layering due to gravity with the horizontal one due to tectonic stresses, salt intrusion pressure, or vertical fractures in potential reservoir regions (Schoenberg and Helbig, 1997). The ORT velocity model with vertical and horizontal symmetry planes is an adequate approximation for many geologic models. However, the computation of traveltimes in ORT anisotropic media is not trivial due to the computational complexity of the eikonal equation in such media even for the acoustic case (Alkhalifah, 2003).

To approximate the eikonal in ORT media, we propose to apply perturbation theory with respect to three anelliptic parameters that we assume to be small. A similar approach was earlier applied for a transversely isotropic medium with vertical (Alkhalifah, 2011a) and tilted (Alkhalifah, 2011b; Stovas and Alkhalifah, 2012) symmetry axes. Recently, Masmoudi and Alkhalifah (2016) propose a similar approach with parameterization based on a weak difference between the parameters defined in the vertical symmetry planes. The application of perturbation theory reduces the complexity of computing the traveltime from solving a partial differential equation (PDE) of a sixth-order polynomial in traveltime to a quadratic in traveltime corresponding to an elliptical background, in addition to nine linear first-order PDEs for the perturbation coefficients. These perturbation coefficients represent the sensitivity of the traveltime in ORT media to the perturbation parameters, and thus give insight into the dependency of the data to the different model parameters. For ORT medium, we select two different sets of anelliptic parameters for the analysis. The first one is related to anellipticity function defined at zero offset: $\eta_{1}$ ([xz] symmetry plane), $\eta_{2}$ ([yz] symmetry plane), and $\eta_{x y}$ (the crossterm anelliptic parameter controlling the azimuthal behavior of anellipticity between vertical symmetry planes) (Stovas, 2015). The second one is related to anellipticity parameters defined for all symmetry planes: $\eta_{1}, \eta_{2}$, and $\eta_{3}$ (Tsvankin, 1997). Assuming a homogeneous ORT medium, the perturbation coefficients can be computed analytically, and the perturbation series results in simple and accurate moveout approximation based on the Shanks transform (Bender and Orszag, 1978). The accuracy of the proposed approximation for both parameterizations is tested against the traveltime approximation proposed by Vasconcelos and Tsvankin (2006) using two ORT models. The results show superior accuracy of proposed approximation.

In our approach, for simplicity, we focus on the vertical ORT model only. However, this method can be extended to the tilted ORT

Manuscript received by the Editor 25 February 2016; revised manuscript received 11 June 2016; published online 12 October 2016.

${ }^{1}$ Norwegian University of Science and Technology, Department of Petroleum Engineering and Applied Geophysics, Trondheim, Norway. E-mail: alexey. stovas@ntnu.no.

${ }^{2}$ King Abdullah University of Science and Technology, Department of Earth Science and Engineering, Thuwal, Saudi Arabia. E-mail: nabil.masmoudi@kaust .edu.sa; tariq.alkhalifah@kaust.edu.sa.

(C) 2016 Society of Exploration Geophysicists. All rights reserved. 
medium in a similar way as shown in Hao and Stovas (2016). There are some pitfalls in specifying the orientation of the ORT symmetry planes (Fowler, 2015), but within the frame of our method, it is not important.

\section{THE ORT P-WAVE EIKONAL EQUATION}

An eikonal equation for acoustic P-waves in ORT media with vertical symmetry axis, which governs traveltime in the high-frequency asymptotic approximation, can easily be derived from the slowness surface equation (Alkhalifah, 2003; Stovas, 2015; Masmoudi and Alkhalifah, 2016):

$$
\begin{gathered}
v_{0}^{2}\left(\frac{\partial \tau}{\partial z}\right)^{2}+v_{1}^{2}\left(1+2 \eta_{1}\right)\left(\frac{\partial \tau}{\partial x}\right)^{2}+v_{2}^{2}\left(1+2 \eta_{2}\right)\left(\frac{\partial \tau}{\partial y}\right)^{2} \\
-2 \eta_{1} v_{0}^{2} v_{1}^{2}\left(\frac{\partial \tau}{\partial z}\right)^{2}\left(\frac{\partial \tau}{\partial x}\right)^{2} \\
-2 \eta_{2} v_{0}^{2} v_{2}^{2}\left(\frac{\partial \tau}{\partial z}\right)^{2}\left(\frac{\partial \tau}{\partial y}\right)^{2}-\left(\left(1+2 \eta_{1}\right)\left(1+2 \eta_{2}\right)\right. \\
\left.-\left(1+\eta_{x y}\right)^{2}\right) v_{1}^{2} v_{2}^{2}\left(\frac{\partial \tau}{\partial x}\right)^{2}\left(\frac{\partial \tau}{\partial y}\right)^{2} \\
+v_{0}^{2} v_{1}^{2} v_{2}^{2}\left(4 \eta_{1} \eta_{2}-\eta_{x y}^{2}\right)\left(\frac{\partial \tau}{\partial z}\right)^{2}\left(\frac{\partial \tau}{\partial x}\right)^{2}\left(\frac{\partial \tau}{\partial y}\right)^{2}=1
\end{gathered}
$$

where $\tau(x, y, z)$ is the traveltime measured from the source at the origin to a point in space $(x, y, z) ; v_{0}, v_{1}, v_{2}$ are the vertical $\mathrm{P}$-wave velocity and P-wave NMO velocities defined in vertical symmetry planes $[x, z]$ and $[y, z]$, respectively; and $\eta_{1}, \eta_{2}, \eta_{x y}$ are the anelliptic parameters, where the first two are defined in vertical symmetry planes $[x, z]$ and $[y, z]$, respectively, and the third one is given by Stovas (2015)

$$
\eta_{x y}=\sqrt{\frac{\left(1+2 \eta_{1}\right)\left(1+2 \eta_{2}\right)}{\left(1+2 \eta_{3}\right)}}-1,
$$

where $\eta_{3}$ is an anelliptic parameter defined in horizontal symmetry plane $[x, y]$ (Vasconcelos and Tsvankin, 2006). Note that indices $(1,2)$ for NMO velocities and anellipticity parameters are different from those defined by Tsvankin (1997). The notations for anisotropy parameters are given in Appendix A.

Because no angles are involved in equation 1 , we assume the vertical ORT model, and orientation of the symmetry planes in ORT medium, are not changing with spatial coordinates.

\section{APPROXIMATE SOLUTION}

Assuming that the anellipticity parameters $\eta_{1}, \eta_{2}$, and $\eta_{x y}$ are small, we approximate the traveltime by the following trial solution of the eikonal equation:

$$
\begin{aligned}
& \tau(x, y, z) \approx \tau_{0}(x, y, z)+\sum_{j=1,2,3} a_{j}(x, y, z) \eta_{j} \\
& +\sum_{\substack{j, k=1,2,3 \\
j \leq k}} b_{j k}(x, y, z) \eta_{j} \eta_{k},
\end{aligned}
$$

where index $3 \equiv x y$. A similar perturbation approach is used in Hao and Stovas (2016) to develop an approximation for a slowness surface in tilted ORT media.

Masmoudi and Alkhalifah (2016) use similar perturbation series but using another parameter $\Delta \chi$ instead of $\eta_{3}$. This parameter can be expressed as $\Delta \chi=\left(\sqrt{\left(1+2 \eta_{2}\right) /\left(\left(1+2 \eta_{1}\right)\left(1+2 \eta_{3}\right)\right)}-1\right)\left(v_{2} / v_{1}\right)$. Being compared with $\eta_{x y}$ from equation 2 , one can see that parameter $\Delta \chi$ depends on the specification of vertical symmetry planes and normalized with the NMO velocities. One more difference is that the second-order coefficients related to parameter $\Delta \chi$ are neglected, $b_{13}=b_{23}=b_{33}=0$.

In equation 3 , we introduce 10 coefficients: the zeroth-order (or ellipsoidal) one, three first-order coefficients $a_{j}$, and six second-order coefficients $b_{j k}$. The zeroth-order coefficient $\tau_{0}(x, y, z)$ is the solution of the elliptic eikonal equation

$$
v_{0}^{2}\left(\frac{\partial \tau}{\partial z}\right)^{2}+v_{1}^{2}\left(\frac{\partial \tau}{\partial x}\right)^{2}+v_{2}^{2}\left(\frac{\partial \tau}{\partial y}\right)^{2}=1,
$$

that is, for a homogeneous medium, given by a simple hyperbolic equation,

$$
\tau_{0}(x, y, z)=\sqrt{\frac{x^{2}}{v_{1}^{2}}+\frac{y^{2}}{v_{2}^{2}}+\frac{z^{2}}{v_{0}^{2}}} .
$$

The functions $a_{j}(x, y, z)$ and $b_{j k}(x, y, z)$ can be obtained using the same scheme used in Alkhalifah (2011a) and Stovas and Alkhalifah (2012) as follows. By substituting trial solution 3 into equation 1 and equating the terms for the same order of anelliptic parameters, we can obtain the PDEs for coefficients $a_{j}$ and $b_{j k}$. The first-order coefficients $a_{j}$ are computed from the linear first-order PDEs:

$$
\begin{aligned}
& v_{0}^{2}\left(\frac{\partial \tau_{0}}{\partial z}\right)\left(\frac{\partial a_{j}}{\partial z}\right)+v_{1}^{2}\left(\frac{\partial \tau_{0}}{\partial x}\right)\left(\frac{\partial a_{j}}{\partial x}\right) \\
& \quad+v_{2}^{2}\left(\frac{\partial \tau_{0}}{\partial y}\right)\left(\frac{\partial a_{j}}{\partial y}\right)=f_{i}\left(\frac{\partial \tau_{0}}{\partial x}, \frac{\partial \tau_{0}}{\partial y}, \frac{\partial \tau_{0}}{\partial z}\right),
\end{aligned}
$$

where the right-side functions $f_{j}$ are given by the zeroth-order coefficient $\tau_{0}$ computed from equation 4 :

$$
\begin{aligned}
& f_{1}\left(\frac{\partial \tau_{0}}{\partial x}, \frac{\partial \tau_{0}}{\partial y}, \frac{\partial \tau_{0}}{\partial z}\right)=v_{1}^{2}\left(\frac{\partial \tau_{0}}{\partial x}\right)^{2}\left(-1+v_{0}^{2}\left(\frac{\partial \tau_{0}}{\partial z}\right)^{2}+v_{2}^{2}\left(\frac{\partial \tau_{0}}{\partial y}\right)^{2}\right) . \\
& f_{2}\left(\frac{\partial \tau_{0}}{\partial x}, \frac{\partial \tau_{0}}{\partial y}, \frac{\partial \tau_{0}}{\partial z}\right)=v_{2}^{2}\left(\frac{\partial \tau_{0}}{\partial y}\right)^{2}\left(-1+v_{0}^{2}\left(\frac{\partial \tau_{0}}{\partial z}\right)^{2}+v_{1}^{2}\left(\frac{\partial \tau_{0}}{\partial x}\right)^{2}\right) \\
& f_{3}\left(\frac{\partial \tau_{0}}{\partial x}, \frac{\partial \tau_{0}}{\partial y}, \frac{\partial \tau_{0}}{\partial z}\right)=-v_{1}^{2} v_{2}^{2}\left(\frac{\partial \tau_{0}}{\partial x}\right)^{2}\left(\frac{\partial \tau_{0}}{\partial y}\right)^{2}
\end{aligned}
$$

The second-order coefficients are computed from the linear firstorder PDEs given by

$$
\begin{gathered}
v_{0}^{2}\left(\frac{\partial \tau_{0}}{\partial z}\right)\left(\frac{\partial b_{j k}}{\partial z}\right)+v_{1}^{2}\left(\frac{\partial \tau_{0}}{\partial x}\right)\left(\frac{\partial b_{j k}}{\partial x}\right)+v_{2}^{2}\left(\frac{\partial \tau_{0}}{\partial y}\right)\left(\frac{\partial b_{j k}}{\partial y}\right) \\
=g_{j k}\left(\frac{\partial \tau_{0}}{\partial x}, \frac{\partial \tau_{0}}{\partial y}, \frac{\partial \tau_{0}}{\partial z} ; \frac{\partial a_{j, k}}{\partial x}, \frac{\partial a_{j, k}}{\partial y}, \frac{\partial a_{j, k}}{\partial z}\right),
\end{gathered}
$$

where the right-side functions $g_{j k}$ are given by the zeroth-order coefficient $\tau_{0}$ computed from equation 4 and the first-order coefficients 
$a_{j}$ and $a_{k}$ are obtained from equation 6 . The functions $g_{j k}$ are algebraically complicated analytical functions (Appendix B).

Note that equations 6 and 8 are linear first-order PDEs with respect to coefficients $a_{j}$ and $b_{j k}$. Therefore, the proposed approximation suggests solving one second-order PDE (equation 4) and nine first-order PDE (equations 6 and 8) instead of solving one sixth-order PDE (equation 1).

\section{A HOMOGENEOUS MODEL TEST}

In a homogeneous medium, using equation 5, all the coefficients in equation 3 can be computed analytically. The first-order coefficients are given by

$$
a_{1}=-\frac{t_{x}^{4}}{\tau_{0}^{3}}, \quad a_{2}=-\frac{t_{y}^{4}}{\tau_{0}^{3}}, \quad a_{3}=-\frac{t_{x}^{2} t_{y}^{2}}{\tau_{0}^{3}}
$$

and the second-order coefficients result in the following solutions:

$$
\begin{aligned}
& b_{11}=\frac{3 t_{x}^{6}\left(\tau_{0}^{2}+3\left(t_{y}^{2}+t_{0}^{2}\right)\right)}{2 \tau_{0}^{7}}, b_{12}=-\frac{9 t_{x}^{4} t_{y}^{4}}{\tau_{0}^{7}}, b_{13}=\frac{3 t_{x}^{4} t_{y}^{2}\left(2 \tau_{0}^{2}-3 t_{x}^{2}\right)}{\tau_{0}^{7}} \\
& b_{22}=\frac{3 t_{y}^{6}\left(\tau_{0}^{2}+3\left(t_{x}^{2}+t_{0}^{2}\right)\right)}{2 \tau_{0}^{7}}, b_{23}=\frac{3 t_{x}^{2} t_{y}^{4}\left(2 \tau_{0}^{2}-3 t_{y}^{2}\right)}{\tau_{0}^{7}} \\
& b_{33}=\frac{3 t_{x}^{2} t_{y}^{2}\left(\tau_{0}^{2}\left(\tau_{0}^{2}-t_{0}^{2}\right)-3 t_{x}^{2} t_{y}^{2}\right)}{2 \tau_{0}^{7}}
\end{aligned}
$$

where $t_{x}^{2}=x^{2} / v_{1}^{2}, t_{y}^{2}=y^{2} / v_{2}^{2}$, and $t_{0}^{2}=z^{2} / v_{0}^{2}$ with velocities being defined after equation 1 .

These coefficients can be considered as sensitivity coefficients for traveltimes in ORT media. They can be used for a simple traveltime approximation for ORT media based on the Shanks transform (Bender and Orszag, 1978):

$$
t(x, y)=\frac{A_{0} A_{2}-A_{1}^{2}}{A_{0}-2 A_{1}+A_{2}},
$$

with

$$
\begin{aligned}
& A_{0}=\tau_{0}\left(x, y, z=v_{0} t_{0}\right), \\
& A_{1}=A_{0}+\sum_{j=1,2,3} a_{j}\left(x, y, z=v_{0} t_{0}\right) \eta_{j}, \\
& A_{2}=A_{1}+\sum_{\substack{j, k=1,2,3 \\
j \leq k}} b_{j k}\left(x, y, z=v_{0} t_{0}\right) \eta_{j} \eta_{k} .
\end{aligned}
$$

Note that along one of the symmetry planes, the coefficients 9 and 10, and approximations 11 and 12 reduce to the ones derived in Alkhalifah (2011a).

\section{ALTERNATIVE PARAMETERIZATION}

The problem described above can also be formulated in terms of another set of anellipticity parameters, specifically in terms of $\eta_{1}$, $\eta_{2}$, and $\eta_{3}$. In this case, the trial solution takes the following form:

$$
\begin{aligned}
& \tau(x, y, z) \approx \tau_{0}(x, y, z)+\sum_{j=1,2,3} a_{j}^{\prime}(x, y, z) \eta_{j} \\
& +\sum_{\substack{j, k=1,2,3 \\
j \leq k}} b_{j k}^{\prime}(x, y, z) \eta_{j} \eta_{k},
\end{aligned}
$$

where index 3 corresponds to parameter $\eta_{3}$. To obtain the coefficients in equation 13 , we expand equation 2 in terms of $\eta_{1}, \eta_{2}$, and $\eta_{3}$ up to second order:

$\eta_{x y}=\eta_{1}+\eta_{2}-\eta_{3}-\frac{1}{2} \eta_{1}^{2}-\frac{1}{2} \eta_{2}^{2}+\frac{3}{2} \eta_{3}^{2}+\eta_{1} \eta_{2}-\eta_{1} \eta_{3}-\eta_{2} \eta_{3}+\cdots$.

By using equation 14, we can rearrange the functions $f_{j}$ and $g_{j k}$ in equations 6 and 8. Considering a homogeneous model, equation 13 results in new first-order coefficients $a_{j}^{\prime}$, which can be written as a linear combination of the coefficients defined in equation 9:

$$
\begin{aligned}
& a_{1}^{\prime}=a_{1}+a_{3}=-\frac{t_{x}^{2}\left(t_{x}^{2}+t_{y}^{2}\right)}{\tau_{0}^{3}}, \\
& a_{2}^{\prime}=a_{2}+a_{3}=-\frac{t_{x}^{2}\left(t_{x}^{2}+t_{y}^{2}\right)}{\tau_{0}^{3}}, \\
& a_{3}^{\prime}=-a_{3}=\frac{t_{x}^{2} t_{y}^{2}}{\tau_{0}^{3}} .
\end{aligned}
$$

The second-order coefficients $b_{j k}^{\prime}$ are given by

$$
\begin{aligned}
b_{11}^{\prime}= & b_{11}+b_{13}+b_{33}-\frac{1}{2} a_{3} \\
& =\frac{t_{x}^{2}\left(t_{0}^{4} t_{y}^{2}+t_{0}^{2}\left(t_{x}^{2}+t_{y}^{2}\right)\left(12 t_{x}^{2}+5 t_{y}^{2}\right)+\left(t_{x}^{2}+t_{y}^{2}\right)^{2}\left(3 t_{x}^{2}+4 t_{y}^{2}\right)\right)}{2 \tau_{0}^{7}} \\
b_{22}^{\prime} & =b_{22}+b_{23}+b_{33}-\frac{1}{2} a_{3} \\
& =\frac{t_{y}^{2}\left(t_{0}^{4} t_{x}^{2}+t_{0}^{2}\left(t_{x}^{2}+t_{y}^{2}\right)\left(5 t_{x}^{2}+12 t_{y}^{2}\right)+\left(t_{x}^{2}+t_{y}^{2}\right)^{2}\left(4 t_{x}^{2}+3 t_{y}^{2}\right)\right)}{2 \tau_{0}^{7}}, \\
b_{33}^{\prime}= & b_{33}+\frac{3}{2} a_{3}=-\frac{t_{x}^{2} t_{y}^{2}\left(t_{0}^{4}+t_{0}^{2}\left(t_{x}^{2}+t_{y}^{2}\right)+3 t_{x}^{2} t_{y}^{2}\right)}{2 \tau_{0}^{7}} \\
b_{12}^{\prime}= & b_{12}+b_{13}+b_{23}+2 b_{33}+a_{3} \\
& =-\frac{t_{x}^{2} t_{y}^{2}\left(t_{0}^{4}-7 t_{0}^{2}\left(t_{x}^{2}+t_{y}^{2}\right)+\left(t_{x}^{2}+t_{y}^{2}\right)^{2}\right)}{\tau_{0}^{7}}, \\
b_{13}^{\prime}= & -b_{13}-2 b_{33}-a_{3} \\
& =\frac{t_{x}^{2} t_{y}^{2}\left(t_{0}^{4}-t_{0}^{2}\left(7 t_{x}^{2}+t_{y}^{2}\right)+\left(t_{x}^{2}+t_{y}^{2}\right)\left(t_{x}^{2}-2 t_{y}^{2}\right)\right)}{\tau_{0}^{7}}, \\
b_{23}^{\prime}= & -b_{23}-2 b_{33}-a_{3} \\
& =\frac{t_{x}^{2} t_{y}^{2}\left(t_{0}^{4}-t_{0}^{2}\left(t_{x}^{2}+7 t_{y}^{2}\right)+\left(t_{x}^{2}+t_{y}^{2}\right)\left(-2 t_{x}^{2}+t_{y}^{2}\right)\right)}{(16)} .
\end{aligned}
$$

Note that equations for coefficients $a_{j}^{\prime}$ and $b_{j k}^{\prime}$ are more complicated compared with coefficients $a_{j}$ and $b_{j k}$. The Shanks transforms 11 and 12 can be defined in a similar manner using the new coefficients $a_{j}^{\prime}$ (equation 15) and $b_{j k}^{\prime}$ (equation 16) substituted into equation 13. 


\section{NUMERICAL EXAMPLES}

To illustrate the sensitivity of the traveltime to all the anelliptic parameters $\left(\eta_{1}, \eta_{2}\right.$, and $\left.\eta_{x y}\right)$, we plot the coefficients $a_{j}$ (Figure 1) and $b_{j k}$ (Figure 2) versus (offsets to depth rations) $x / z$ and $y / z$ for ORT model with velocities $v_{0}=2 \mathrm{~km} / \mathrm{s}, v_{1}=2.2 \mathrm{~km} / \mathrm{s}$, and $v_{2}=2.5 \mathrm{~km} / \mathrm{s}$. This set of velocities results in rather large values for anisotropic parameters $\delta_{1}$ and $\delta_{2}$; however, we select these numbers to illustrate nonsymmetric behavior of sensitivity parameters. We can see that the magnitude of the first-order coefficients $a_{1}$ and $a_{2}$ are larger than the magnitude of the coefficient $a_{3}$. A similar behavior happens for the second-order coefficients $b_{11}, b_{22}$, and $b_{33}$ (Figure 2a and 2c). The crossterm second-order coefficients $b_{12}, b_{13}$, and $b_{23}$ (Figure $2 \mathrm{~b}$ and $2 \mathrm{~d}$ ) are smaller compared with coefficients $b_{11}$ and $b_{22}$. The first-order coefficients $a_{1}^{\prime}, a_{2}^{\prime}$, and $a_{3}^{\prime}$ computed for parameterization $\left(\eta_{1}, \eta_{2}\right.$, and $\left.\eta_{3}\right)$ are shown in Figure 3. One can see that they are slightly different from their counterparts shown in Figure 1. The second-order coefficients $b_{11}^{\prime}, b_{22}^{\prime}$, and $b_{33}^{\prime}$ (Figure $4 \mathrm{a}$ and $4 \mathrm{c}$ ) and $b_{12}^{\prime}, b_{13}^{\prime}$, and $b_{23}^{\prime}$ (Figure $4 \mathrm{~b}$ and $4 d$ ) are very different from their counterparts shown in Figure 2. In particular, we notice anomalies in coefficient $b_{12}^{\prime}$, whereas coefficient $b_{12}$ possesses a very smooth behavior. These anomalies are the result of crosstalk between the crossterm coefficient $b_{12}$ and coefficients $a_{3}$ and $b_{33}$ (equation 16).

To test the accuracy of the proposed traveltime approximations 11 and 12 with the different parameterizations in anelliptic parameters $\left(\eta_{1}, \eta_{2}\right.$, and $\left.\eta_{x y}\right)$ and $\left(\eta_{1}, \eta_{2}\right.$, and $\left.\eta_{3}\right)$, we choose two ORT models with vertical and NMO velocities mentioned above, anelliptic parameters $\eta_{1}=0.15, \eta_{2}=0.1$, and two different parameters $\eta_{3}=0.2\left(\eta_{x y}=0.056\right)$ and $\eta_{3}=-0.1\left(\eta_{x y}=0.396\right)$. The choice of these models were made to highlight the accuracy of the two parameterizations with respect to the choice of the third anellipticity parameter, mainly controlled by magnitude of crossterm anelliptic parameter $\eta_{x y}$. To illustrate the anisotropy behavior of the two mod- a)

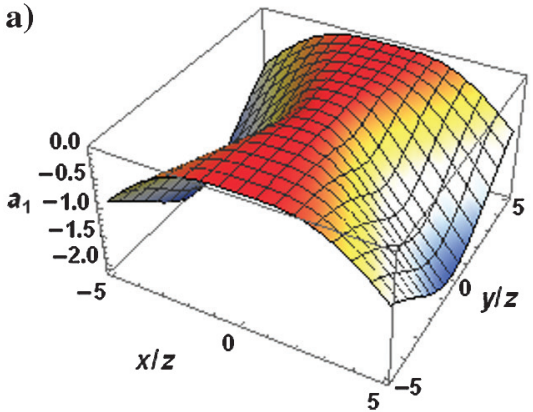

b)

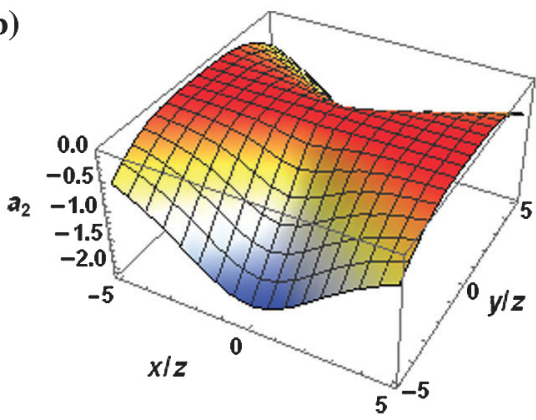

c)

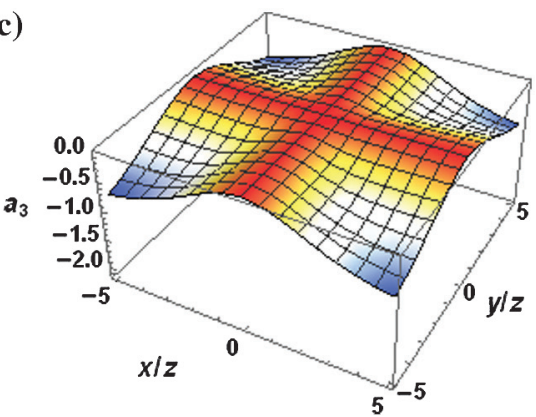

Figure 1. (a-c) The first-order coefficients $a_{1}, a_{2}$, and $a_{3}$ plotted versus offset/depth projections. The model is defined by three velocities $v_{0}=2 \mathrm{~km} / \mathrm{s}, v_{1}=2.2 \mathrm{~km} / \mathrm{s}$, and $v_{2}=2.5 \mathrm{~km} / \mathrm{s}$.

a)

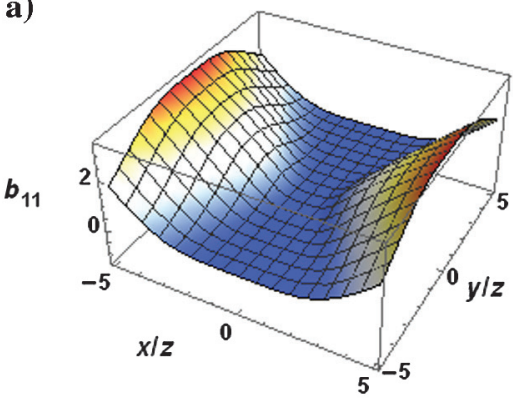

d)

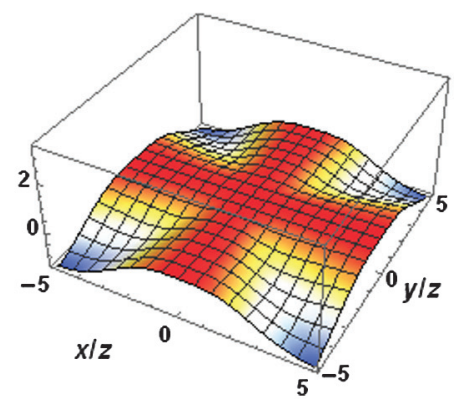

b)

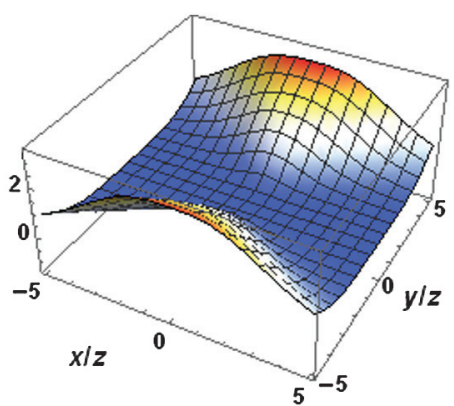

e)

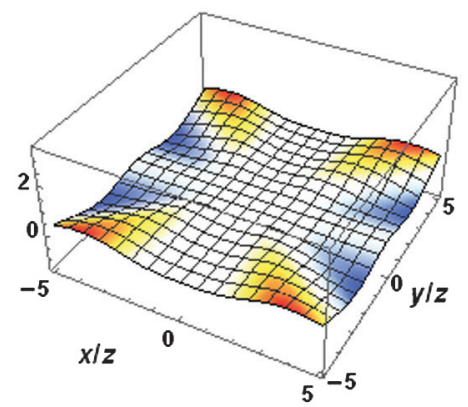

c)

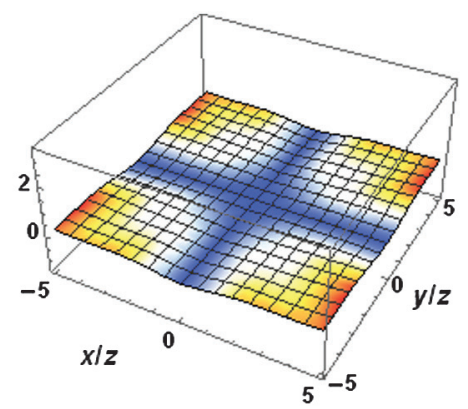

f)

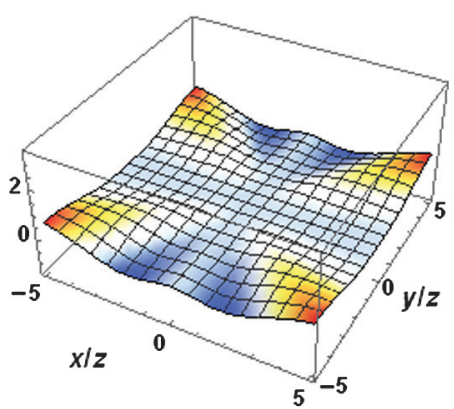

Figure 2. (a-c) The second-order coefficients $b_{11}, b_{22}$, and $b_{33}$ and (d-f) $b_{12}, b_{13}$, and $b_{23}$ plotted versus offset/depth projections. The model is defined by three velocities $v_{0}=2 \mathrm{~km} / \mathrm{s}, v_{1}=2.2 \mathrm{~km} / \mathrm{s}$, and $v_{2}=2.5 \mathrm{~km} / \mathrm{s}$. 
els, we plot the NMO ellipse and two anellipticities (Stovas, 2015) in polar plots. Models 1 and 2 have the same NMO ellipse (Figure 5a), but different anellipticities (Figure 5b). One can see that model 2 is more anelliptical than model 1 . The accuracy plots from approximations 11 and 12 with the two parameterizations of the anelliptic parameters applied for models 1 and 2 are shown in Figure 6. The exact traveltime is computed from parametric equations for P-waves in acoustic ORT media (Stovas, 2015).

For model 1 with weak crossterm anelliptic parameter $\eta_{x y}$, the accuracy of the traveltime approximations 11 and 12 is very similar for these parameterizations in anelliptic parameters (Figure 6a and $6 c)$. However, for model 2 with strong crossterm anelliptic parameter $\eta_{x y}$, the accuracy of the approximation based on the parameterization with $\eta_{x y}$ is better (Figure $6 \mathrm{~b}$ and $6 \mathrm{~d}$ ). For both models, the relative error in traveltime is less than $0.1 \%$ regardless to the choice of parameterization. To compare the accuracy, we also perform the computation with the traveltime approximation proposed by Vasconcelos and Tsvankin (2006). The results are shown in Figure 7.
One can see that for both models, the accuracy of our approximation is much better.

\section{DISCUSSION}

The sensitivity coefficients $a_{1}$ and $a_{2}$ equal zero in mutually orthogonal vertical symmetry planes, $a_{1}=0$ when $x=0$, and $a_{2}=$ 0 when $y=0$. The sensitivity coefficient $a_{3}=0$ when $x=0$ or $y=0$. However, between the symmetry planes, $x, y \neq 0$, these coefficients do not equal zero. Ideally, the azimuthal dependence of coefficients $a_{1}$ and $a_{2}$ should be mostly focused with the corresponding symmetry plane, whereas the azimuthal dependence of coefficient $a_{3}$ should be mostly focused at azimuth of approximately $\theta=\pi / 4$, with as little overlapping as possible.

This is important for inversion of anelliptic parameters from traveltime data. The overlapping of polar graphs for sensitivity coefficients indicates the azimuth range with similar influence of
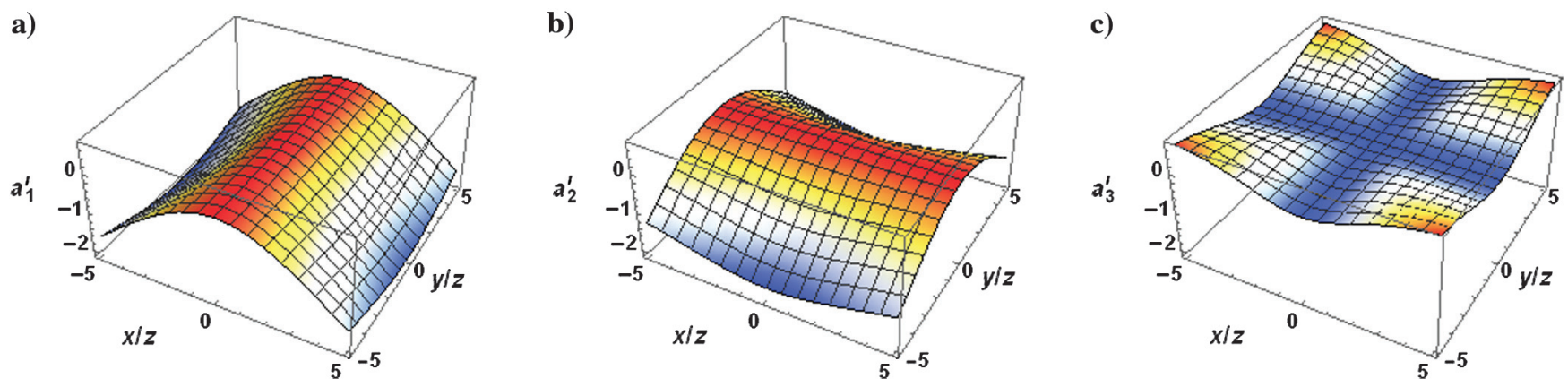

Figure 3. (a-c) The first-order coefficients $a_{1}^{\prime}, a_{2}^{\prime}$, and $a_{3}^{\prime}$ plotted versus offset/depth projections. The model is defined by three velocities $v_{0}=2 \mathrm{~km} / \mathrm{s}, v_{1}=2.2 \mathrm{~km} / \mathrm{s}$, and $v_{2}=2.5 \mathrm{~km} / \mathrm{s}$.
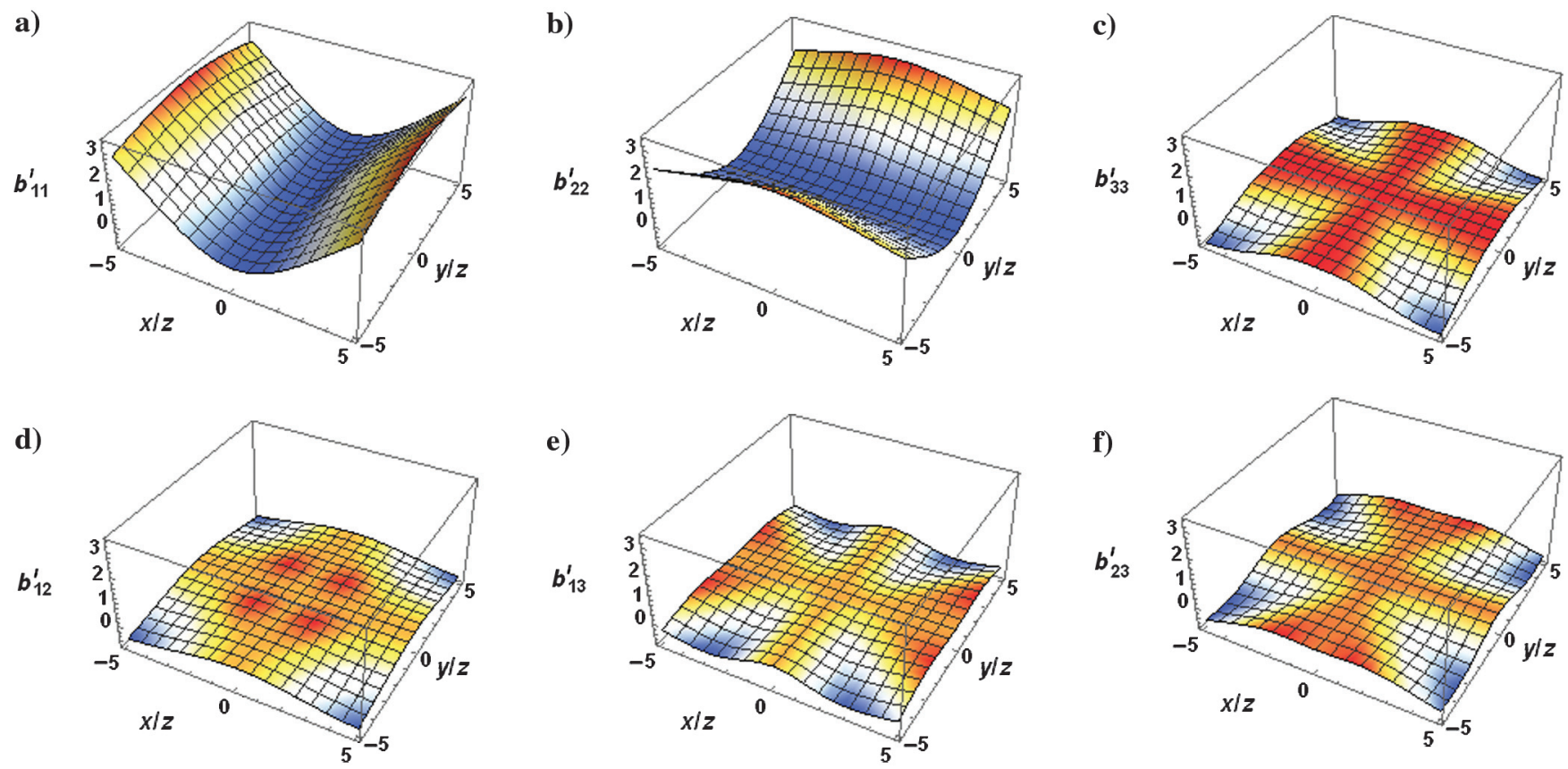

Figure 4. (a-c) The second-order coefficients $b_{11}^{\prime}, b_{22}^{\prime}$, and $b_{33}^{\prime}$ and (d-f) $b_{12}^{\prime}, b_{13}^{\prime}$, and $b_{23}^{\prime}$ plotted versus offset/depth projections. The model is defined by three velocities $v_{0}=2 \mathrm{~km} / \mathrm{s}, v_{1}=2.2 \mathrm{~km} / \mathrm{s}$, and $v_{2}=2.5 \mathrm{~km} / \mathrm{s}$. 
annelliptic parameters on traveltime, which can be considered as the azimuthal crosstalk between these parameters.

The crosstalk between anelliptic parameters $\eta_{1}$ and $\eta_{2}$ with different parameterizations discussed above can be illustrated by plotting coefficients $a_{j}$ and $a_{j}^{\prime}\left(b_{j k}\right.$ and $\left.b_{j k}^{\prime}\right)$ in polar plots showing their azi- muthal dependence. To do that, we substitute lateral coordinates with $x=r \cos \theta$ and $y=r \sin \theta$, where $r$ is the radial offset and $\theta$ is the azimuth. For given $t_{0}=1 \mathrm{~s}, r=5 \mathrm{~km}$, and velocities $v_{1}=2.2 \mathrm{~km} / \mathrm{s}$ and $v_{2}=2.5 \mathrm{~km} / \mathrm{s}$, we plot the coefficients $a_{j}$ and $a_{j}^{\prime}\left(b_{j k}\right.$ and $\left.b_{j k}^{\prime}\right)$ in Figure 8. The first-order coefficients from both
Figure 5. The NMO ellipse (a) and anellipticity (b) for ORT models 1 and 2 . a)

Figure 6. The relative error (in \%) in traveltime from equations 11 and 12 plotted versus offset/depth projections. Two parameterizations $\left(\eta_{1}, \eta_{2}\right.$ and $\left.\eta_{x y}\right)$ and $\left(\eta_{1}, \eta_{2}\right.$ and $\left.\eta_{3}\right)$ are used in computation for two models with three velocities $v_{0}=2 \mathrm{~km} / \mathrm{s}$, $v_{1}=2.2 \mathrm{~km} / \mathrm{s}$, and $v_{2}=2.5 \mathrm{~km} / \mathrm{s}$, anellipticity parameters $\eta_{1}=0.15$ and $\eta_{2}=0.1$ with $\eta_{3}=0.2$ $\left(\eta_{x y}=0.056\right)$ for model 1 and $\eta_{3}=-0.1\left(\eta_{x y}=\right.$ $0.396)$ for model 2 . The results with first parameterization applied for models 1 and 2 are shown in (a) and (c), respectively. The results with second parameterization applied for models 1 and 2 are shown in (b) and (d), respectively. Figure 7. The relative error (in \%) in traveltime (2006) plotted versus offset/depth projections. The model is defined by three velocities $v_{0}=2 \mathrm{~km} / \mathrm{s}$, $v_{1}=2.2 \mathrm{~km} / \mathrm{s}, \quad v_{2}=2.5 \mathrm{~km} / \mathrm{s}$ and anellipticity parameters (a) $\eta_{1}=0.15, \eta_{2}=0.1$, and $\eta_{3}=0.2$ $\left(\eta_{x y}=0.056\right)$ and (b) $\eta_{3}=-0.1\left(\eta_{x y}=0.396\right)$.

c)

a)

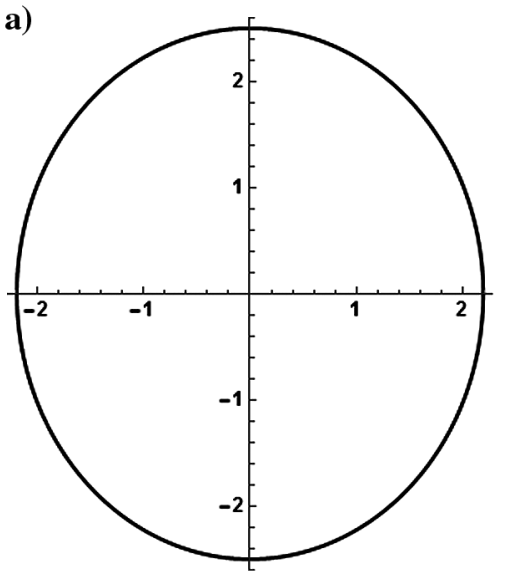

b)
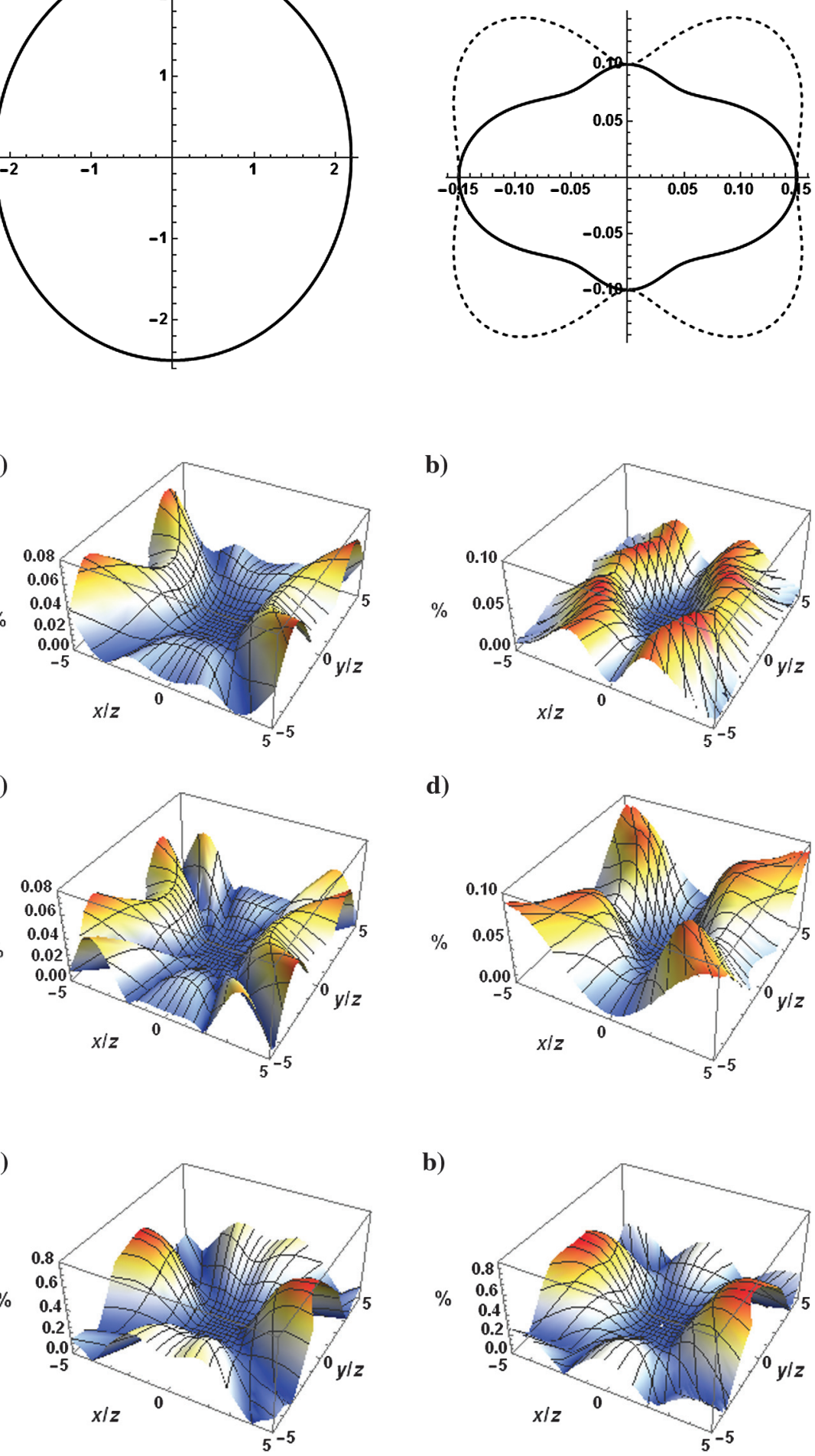

b)

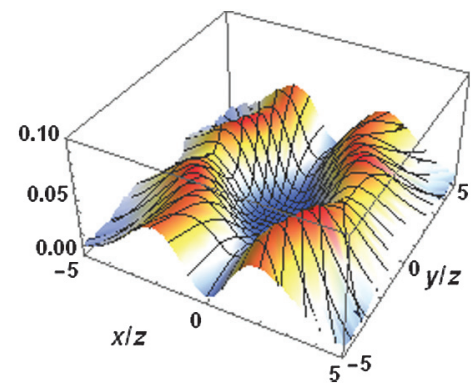

d)

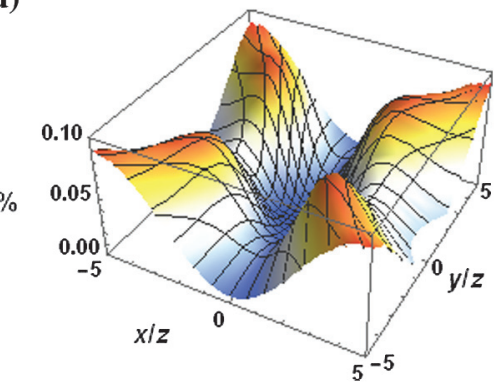

b)

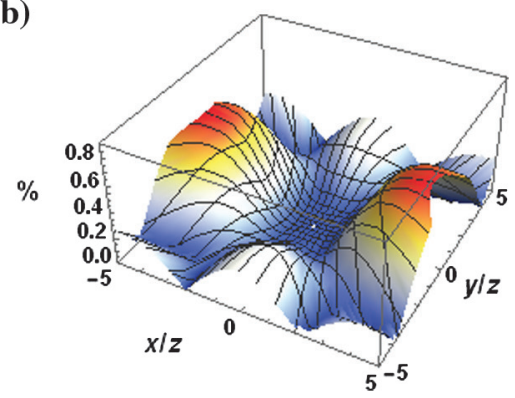


a)

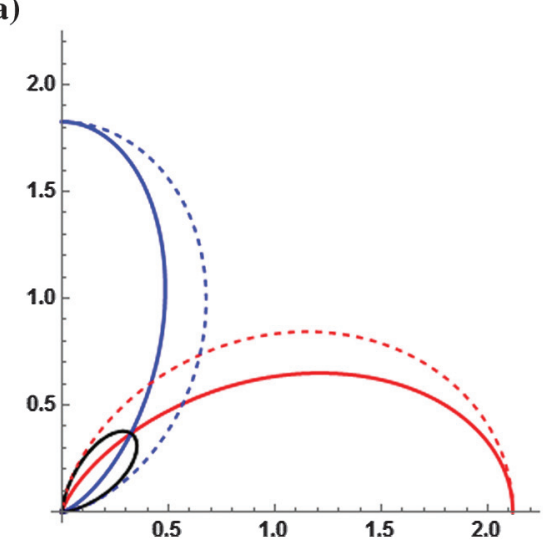

b)

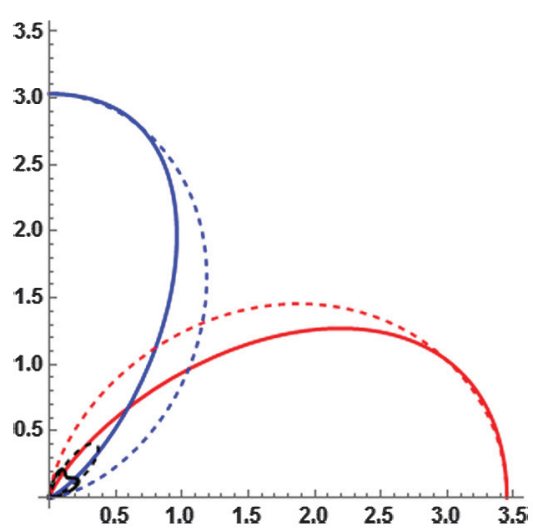

c)

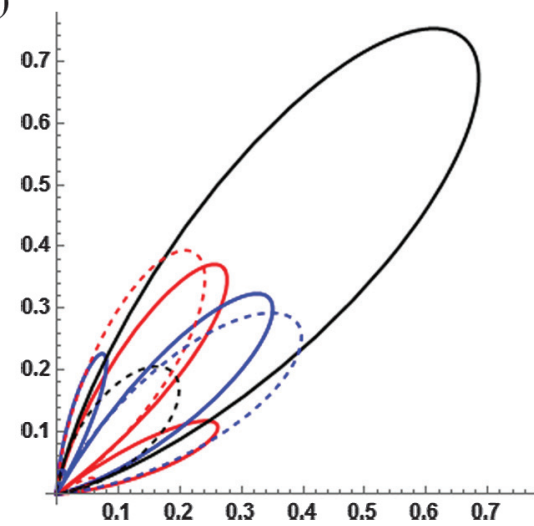

Figure 8. (a) The absolute values of the first-order coefficients $a_{1}$ (red), $a_{2}$, (blue), and $a_{3}$ (black) are shown by solid lines, and $a_{1}^{\prime}$ (red), $a_{2}^{\prime}$ (blue), and $a_{3}^{\prime}$ (black) are shown by dashed lines computed from different parameterizations and plotted in a polar plot. (b) The absolute values of the second-order coefficients $b_{11}$ (red), $b_{22}$ (blue), and $b_{12}$ (black) are shown by solid lines, and $b_{11}^{\prime}$ (red), $b_{22}^{\prime}$ (blue), and $b_{12}^{\prime}$ (black) are shown by dashed lines computed from different parameterizations and plotted in a polar plot. (c) The absolute values of the second-order coefficients $b_{13}$ (red), $b_{23}$ (blue), and $b_{33}$ (black) are shown by solid lines, and $b_{13}^{\prime}$ (red), $b_{23}^{\prime}$ (blue), and $b_{33}^{\prime}$ (black) are shown by dashed lines computed from different parameterizations and plotted in a polar plot. The model is defined by three velocities $v_{0}=2 \mathrm{~km} / \mathrm{s}, v_{1}=2.2 \mathrm{~km} / \mathrm{s}, v_{2}=2.5 \mathrm{~km} / \mathrm{s}$, and radial offset is $5 \mathrm{~km}$.

parameterizations are shown in Figure 8a. The crosstalk between anelliptic parameters $\eta_{1}$ and $\eta_{2}$ can be seen as the overlapping area from $a_{1}$ and $a_{2}\left(a_{1}^{\prime}\right.$ and $\left.a_{2}^{\prime}\right)$. One can see that parameterization with $\eta_{x y}$ results in a smaller overlapping area compared with the one obtained from parameterization with $\eta_{3}$. A similar result can be observed from comparison of second-order coefficients $b_{11}$ and $b_{22}$ $\left(b_{11}^{\prime}\right.$ and $b_{22}^{\prime}$ ), as is shown in Figure 8b. Both crossterm the second-order coefficients $b_{12}$ and $b_{12}^{\prime}$ are oriented for an azimuth of approximately $\theta=\pi / 4$. The behavior of second-order coefficients $b_{13}\left(b_{13}^{\prime}\right)$ and $b_{23}\left(b_{23}^{\prime}\right)$ is more complicated (Figure $8 \mathrm{c}$ ). They have two petals, but $b_{13}$ and $b_{23}$ are less detached to corresponding axes compared with $b_{13}^{\prime}$ and $b_{23}^{\prime}$ indicating less influence of $\eta_{1}$ and $\eta_{2}$ when estimating $\eta_{x y}$.

\section{CONCLUSION}

We have developed the traveltime perturbation method for the acoustic ORT eikonal equation. The perturbation coefficients are used to analyze the sensitivity of the traveltime to anelliptic parameters. This analysis reveals that traveltime is more sensitive to $\eta_{1}$ and $\eta_{2}$ within $[x z]$ and $[y z]$ symmetry planes, and sensitive to $\eta_{x y}$ at a lesser degree mainly along the $45^{\circ}$ azimuth direction. For a homogeneous ORT medium, this method results in simple and accurate moveout approximation. We also developed the perturbation solution for another parameterization in anelliptic parameters in terms of $\eta_{1}, \eta_{2}$, and $\eta_{3}$. The comparison between two parameterizations shows that for models with weak crossterm anelliptic parameter $\eta_{x y}$, both approximations result in similar accuracy, whereas for models with strong $\eta_{x y}$, the parameterization with $\eta_{x y}$ gives better results than the one using $\eta_{3}$. The comparison made with alternative traveltime approximation derived under assumption of azimuthally dependent VTI medium shows that our approximation is much more accurate for both tested ORT models. The analysis of crosstalk between $\eta_{1}$ and $\eta_{2}$ shows that parameterization with $\eta_{x y}$ gives better discrimination between anelliptic parameters.

\section{ACKNOWLEDGMENTS}

A. Stovas would like to acknowledge the ROSE Project for financial support. N. Masmoudi and T. Alkhalifah would like to thank KAUST for financial support.

\section{APPENDIX A}

\section{ORT ANISOTROPY PARAMETER NOTATIONS}

In this paper, the following notations for anisotropy parameters are used. Note that they are slightly different from commonly used notations from Tsvankin (1997). The NMO velocities defined in vertical $[x z]$ and $[y z]$ symmetry planes are, respectively, given by

$$
\begin{aligned}
& v_{1}^{2}=v_{0}^{2}\left(1+2 \delta_{1}\right), \\
& v_{2}^{2}=v_{0}^{2}\left(1+2 \delta_{2}\right) .
\end{aligned}
$$

The anellipticity parameters defined in vertical $[x z]$ and $[y z]$ symmetry planes are, respectively, given by

$$
\begin{aligned}
& \eta_{1}=\frac{\varepsilon_{1}-\delta_{1}}{1+2 \delta_{1}}, \\
& \eta_{2}=\frac{\varepsilon_{2}-\delta_{2}}{1+2 \delta_{2}},
\end{aligned}
$$

and the anellipticity parameter defined in horizontal symmetry plane $[x y]$ is

$$
\eta_{3}=\frac{\varepsilon_{2}-\varepsilon_{1}-\delta_{3}\left(1+2 \varepsilon_{1}\right)}{\left(1+2 \delta_{3}\right)\left(1+2 \varepsilon_{1}\right)}
$$

The anisotropy parameters $\varepsilon_{1}$ and $\varepsilon_{2}$ used in equations above are given by 


$$
\begin{aligned}
& \varepsilon_{1}=\frac{c_{11}-c_{33}}{2 c_{33}}, \\
& \varepsilon_{2}=\frac{c_{22}-c_{33}}{2 c_{33}} .
\end{aligned}
$$

The anisotropy parameters $\delta_{1}, \delta_{2}$, and $\delta_{3}$ used in equations above are given by

$$
\begin{aligned}
& \delta_{1}=\frac{\left(c_{13}+c_{55}\right)^{2}-\left(c_{33}-c_{55}\right)^{2}}{2 c_{33}\left(c_{33}-c_{55}\right)}, \\
& \delta_{2}=\frac{\left(c_{23}+c_{44}\right)^{2}-\left(c_{33}-c_{44}\right)^{2}}{2 c_{33}\left(c_{33}-c_{44}\right)}, \\
& \delta_{3}=\frac{\left(c_{12}+c_{66}\right)^{2}-\left(c_{11}-c_{66}\right)^{2}}{2 c_{11}\left(c_{11}-c_{66}\right)} .
\end{aligned}
$$

The stiffness coefficients in equations A-4 and A-5 are annotated as $c_{i j}$.

\section{APPENDIX B \\ RIGHT-SIDE COEFFICIENTS $g_{j k}$ FROM EQUATION 8}

In the case of a heterogeneous medium, the right-side coefficients $g_{j k}$ in equation 8 can explicitly be written in terms of the zerothorder coefficient and first-order coefficients computed in equations 4 and 6, respectively. On-diagonal second-order coefficients $g_{j j}$ are given by

$$
\begin{gathered}
2 g_{11}\left(\frac{\partial \tau_{0}}{\partial x}, \frac{\partial \tau_{0}}{\partial y}, \frac{\partial \tau_{0}}{\partial z} ; \frac{\partial a_{1}}{\partial x}, \frac{\partial a_{1}}{\partial y}, \frac{\partial a_{1}}{\partial z}\right)=-v_{0}^{2}\left(\frac{\partial a_{1}}{\partial z}\right)^{2} \\
-v_{1}^{2}\left(\frac{\partial a_{1}}{\partial x}\right)\left(4 \frac{\partial \tau_{0}}{\partial x}+\frac{\partial a_{1}}{\partial x}\right)-v_{2}^{2}\left(\frac{\partial a_{1}}{\partial y}\right)^{2} \\
+4 v_{0}^{2} v_{1}^{2} \frac{\partial \tau_{0}}{\partial x} \frac{\partial \tau_{0}}{\partial z}\left(\frac{\partial \tau_{0}}{\partial z} \frac{\partial a_{1}}{\partial x}+\frac{\partial \tau_{0}}{\partial x} \frac{\partial a_{1}}{\partial z}\right) \\
+4 v_{1}^{2} v_{2}^{2} \frac{\partial \tau_{0}}{\partial x} \frac{\partial \tau_{0}}{\partial y}\left(\frac{\partial \tau_{0}}{\partial y} \frac{\partial a_{1}}{\partial x}+\frac{\partial \tau_{0}}{\partial x} \frac{\partial a_{1}}{\partial y}\right)
\end{gathered}
$$

$$
\begin{aligned}
& 2 g_{22}\left(\frac{\partial \tau_{0}}{\partial x}, \frac{\partial \tau_{0}}{\partial y}, \frac{\partial \tau_{0}}{\partial z} ; \frac{\partial a_{2}}{\partial x}, \frac{\partial a_{2}}{\partial y}, \frac{\partial a_{2}}{\partial z}\right)=-v_{0}^{2}\left(\frac{\partial a_{2}}{\partial z}\right)^{2} \\
& -v_{1}^{2}\left(\frac{\partial a_{2}}{\partial x}\right)^{2}-v_{2}^{2}\left(\frac{\partial a_{2}}{\partial y}\right)\left(4 \frac{\partial \tau_{0}}{\partial y}+\frac{\partial a_{2}}{\partial y}\right) \\
& +4 v_{0}^{2} v_{2}^{2} \frac{\partial \tau_{0}}{\partial y} \frac{\partial \tau_{0}}{\partial z}\left(\frac{\partial \tau_{0}}{\partial z} \frac{\partial a_{2}}{\partial y}+\frac{\partial \tau_{0}}{\partial y} \frac{\partial a_{2}}{\partial z}\right) \\
& +4 v_{1}^{2} v_{2}^{2} \frac{\partial \tau_{0}}{\partial x} \frac{\partial \tau_{0}}{\partial y}\left(\frac{\partial \tau_{0}}{\partial y} \frac{\partial a_{2}}{\partial x}+\frac{\partial \tau_{0}}{\partial x} \frac{\partial a_{2}}{\partial y}\right)
\end{aligned}
$$

$$
\begin{aligned}
2 g_{33} & \left(\frac{\partial \tau_{0}}{\partial x}, \frac{\partial \tau_{0}}{\partial y}, \frac{\partial \tau_{0}}{\partial z} ; \frac{\partial a_{3}}{\partial x}, \frac{\partial a_{3}}{\partial y}, \frac{\partial a_{3}}{\partial z}\right)=-v_{0}^{2}\left(\frac{\partial a_{3}}{\partial z}\right)^{2} \\
& -v_{1}^{2}\left(\frac{\partial a_{3}}{\partial x}\right)^{2}-v_{2}^{2}\left(\frac{\partial a_{3}}{\partial y}\right) \\
+ & v_{1}^{2} v_{2}^{2} \frac{\partial \tau_{0}}{\partial x} \frac{\partial \tau_{0}}{\partial y}\left(\frac{\partial \tau_{0}}{\partial x} \frac{\partial \tau_{0}}{\partial y}+4\left(\frac{\partial \tau_{0}}{\partial x} \frac{\partial a_{3}}{\partial y}+\frac{\partial \tau_{0}}{\partial y} \frac{\partial a_{3}}{\partial x}\right)\right) \\
- & v_{0}^{2} v_{1}^{2} v_{2}^{2}\left(\frac{\partial \tau_{0}}{\partial x}\right)^{2}\left(\frac{\partial \tau_{0}}{\partial y}\right)^{2}\left(\frac{\partial \tau_{0}}{\partial z}\right)^{2} .
\end{aligned}
$$

Off-diagonal second-order coefficients $g_{j k}, k>j$ are slightly more complicated

$$
\begin{gathered}
g_{12}\left(\frac{\partial \tau_{0}}{\partial x}, \frac{\partial \tau_{0}}{\partial y}, \frac{\partial \tau_{0}}{\partial z} ; \frac{\partial a_{1,2}}{\partial x}, \frac{\partial a_{1,2}}{\partial y}, \frac{\partial a_{1,2}}{\partial z}\right)=-v_{0}^{2}\left(\frac{\partial a_{1}}{\partial z}\right)\left(\frac{\partial a_{2}}{\partial z}\right) \\
-v_{1}^{2}\left(\frac{\partial a_{2}}{\partial x}\right)\left(2 \frac{\partial \tau_{0}}{\partial x}+\frac{\partial a_{1}}{\partial x}\right)-v_{2}^{2}\left(\frac{\partial a_{1}}{\partial y}\right)\left(2 \frac{\partial \tau_{0}}{\partial y}+\frac{\partial a_{2}}{\partial x}\right) \\
+2 v_{0}^{2} v_{1}^{2} \frac{\partial \tau_{0}}{\partial x} \frac{\partial \tau_{0}}{\partial z}\left(\frac{\partial \tau_{0}}{\partial z} \frac{\partial a_{2}}{\partial x}+\frac{\partial \tau_{0}}{\partial x} \frac{\partial a_{2}}{\partial z}\right)+2 v_{0}^{2} v_{2}^{2} \frac{\partial \tau_{0}}{\partial z} \frac{\partial \tau_{0}}{\partial y} \\
\quad \times\left(\frac{\partial \tau_{0}}{\partial z} \frac{\partial a_{1}}{\partial y}+\frac{\partial \tau_{0}}{\partial y} \frac{\partial a_{1}}{\partial z}\right)+2 v_{1}^{2} v_{2}^{2} \frac{\partial \tau_{0}}{\partial x} \frac{\partial \tau_{0}}{\partial y} \\
\quad \times\left(\frac{\partial \tau_{0}}{\partial x} \frac{\partial \tau_{0}}{\partial y}+\frac{\partial \tau_{0}}{\partial x}\left(\frac{\partial a_{1}}{\partial y}+\frac{\partial a_{2}}{\partial y}\right)+\frac{\partial \tau_{0}}{\partial y}\left(\frac{\partial a_{1}}{\partial x}+\frac{\partial a_{2}}{\partial x}\right)\right) \\
-2 v_{0}^{2} v_{1}^{2} v_{2}^{2}\left(\frac{\partial \tau_{0}}{\partial x}\right)^{2}\left(\frac{\partial \tau_{0}}{\partial y}\right)^{2}\left(\frac{\partial \tau_{0}}{\partial z}\right)^{2},
\end{gathered}
$$

$$
\begin{aligned}
& g_{13}\left(\frac{\partial \tau_{0}}{\partial x}, \frac{\partial \tau_{0}}{\partial y}, \frac{\partial \tau_{0}}{\partial z} ; \frac{\partial a_{1,3}}{\partial x}, \frac{\partial a_{1,3}}{\partial y}, \frac{\partial a_{1,3}}{\partial z}\right) \\
& =-v_{0}^{2}\left(\frac{\partial a_{1}}{\partial z}\right)\left(\frac{\partial a_{3}}{\partial z}\right)-v_{1}^{2}\left(\frac{\partial a_{3}}{\partial x}\right)\left(2 \frac{\partial \tau_{0}}{\partial x}+\frac{\partial a_{1}}{\partial x}\right) \\
& -v_{2}^{2}\left(\frac{\partial a_{1}}{\partial y}\right)\left(\frac{\partial a_{3}}{\partial y}\right)+2 v_{0}^{2} v_{1}^{2} \frac{\partial \tau_{0}}{\partial x} \frac{\partial \tau_{0}}{\partial z}\left(\frac{\partial \tau_{0}}{\partial z} \frac{\partial a_{3}}{\partial x}+\frac{\partial \tau_{0}}{\partial x} \frac{\partial a_{3}}{\partial z}\right) \\
& -2 v_{1}^{2} v_{2}^{2} \frac{\partial \tau_{0}}{\partial x} \frac{\partial \tau_{0}}{\partial y}\left(\frac{\partial \tau_{0}}{\partial x}\left(\frac{\partial a_{1}}{\partial y}-\frac{\partial a_{3}}{\partial y}\right)+\frac{\partial \tau_{0}}{\partial y}\left(\frac{\partial a_{1}}{\partial x}-\frac{\partial a_{3}}{\partial x}\right)\right),
\end{aligned}
$$

$$
\begin{aligned}
& g_{23}\left(\frac{\partial \tau_{0}}{\partial x}, \frac{\partial \tau_{0}}{\partial y}, \frac{\partial \tau_{0}}{\partial z} ; \frac{\partial a_{2,3}}{\partial x}, \frac{\partial a_{2,3}}{\partial y}, \frac{\partial a_{2,3}}{\partial z}\right) \\
& =-v_{0}^{2}\left(\frac{\partial a_{2}}{\partial z}\right)\left(\frac{\partial a_{3}}{\partial z}\right)-v_{1}^{2}\left(\frac{\partial a_{2}}{\partial x}\right)\left(\frac{\partial a_{3}}{\partial x}\right) \\
& -v_{2}^{2}\left(\frac{\partial a_{3}}{\partial y}\right)\left(2 \frac{\partial \tau_{0}}{\partial y}+\frac{\partial a_{2}}{\partial y}\right)+2 v_{0}^{2} v_{2}^{2} \frac{\partial \tau_{0}}{\partial y} \frac{\partial \tau_{0}}{\partial z}\left(\frac{\partial \tau_{0}}{\partial y} \frac{\partial a_{3}}{\partial z}+\frac{\partial \tau_{0}}{\partial z} \frac{\partial a_{3}}{\partial y}\right) \\
& -2 v_{1}^{2} v_{2}^{2} \frac{\partial \tau_{0}}{\partial x} \frac{\partial \tau_{0}}{\partial y}\left(\frac{\partial \tau_{0}}{\partial y}\left(\frac{\partial a_{2}}{\partial x}-\frac{\partial a_{3}}{\partial x}\right)+\frac{\partial \tau_{0}}{\partial x}\left(\frac{\partial a_{2}}{\partial y}-\frac{\partial a_{3}}{\partial y}\right)\right) .(\mathrm{B}-6)
\end{aligned}
$$

\section{REFERENCES}

Alkhalifah, T., 2003, An acoustic wave equation for orthorhombic anisotropy: Geophysics, 68, 1169-1172, doi: 10.1190/1.1598109. 
Alkhalifah, T, 2011a, Scanning anisotropy parameters in complex media: Geophysics, 76, no. 3, U13-U22, doi: 10.1190/1.3553015.

Alkhalifah, T., 2011b, Traveltime approximations for transversely isotropic media with an inhomogeneous background: Geophysics, 76, no. 3, WA31-WA42, doi: 10.1190/1.3555040.

Bender, C. M., and S. A. Orszag, 1978, Advanced mathematical methods for scientists and engineers: McGraw-Hill.

Fowler, P., 2015, Some pitfalls in orthorhombic parameter estimation: 85th Annual International Meeting, SEG, Expanded Abstracts, 493-497.

Hao, Q., and A. Stovas 2016. P-wave slowness surface approximation for tilted orthorhombic media: Geophysics, 81, no. 3, C99-C112, doi: 10 $.1190 /$ geo2015-0440.1.

Masmoudi, N., and T. Alkhalifah, 2016, Traveltime approximations and parameter estimation for orthorhombic media: Geophysics, 81, no. 4 , C127-C137, doi: 10.1190/geo2015-0367.1.
Schoenberg, M., and K. Helbig, 1997, Orthorhombic media: Modeling elastic wave behavior in a vertically fractured earth: Geophysics, 62, 1954 1974, doi: 10.1190/1.1444297.

Stovas, A., 2015, Azimuthally dependent kinematic properties of orthorhombic media: Geophysics, 80, no. 6, C107-C122, doi: 10.1190/geo2015-0288.1. Stovas, A., and T. Alkhalifah, 2012, A new traveltime approximation in TTI media: Geophysics, 77, no 4, C37-C42, doi: 10.1190/geo2011-0158.1.

Tsvankin, I., 1997, Anisotropic parameters and P-wave velocity for orthorhombic media: Geophysics, 62, 1292-1309, doi: 10.1190/1.1444231.

Vasconcelos, I., and I. Tsvankin, 2006, Non-hyperbolic moveout inversion of wide-azimuth P-wave data for orthorhombic media: Geophysical Prospecting, 54, 535-552, doi: 10.1111/j.1365-2478.2006.00559.x. 